\title{
Aggressive Surgical Management in the Treatment of Diabetic Foot Ulcers
}

\author{
Authors \\ Dr Bammidi Sandeep. (M.S) ${ }^{1}$, Dr P.S. Srinivas, M.S (Gen) ${ }^{2}$ \\ ${ }^{1}$ Malla Reddy Institute of Medical Sciences, Suraram X Roads, Quthubullapur, Telangana, INDIA \\ ${ }^{2}$ Associate Professor, ACSR Medical College, Nellore (A.P), INDIA \\ Corresponding Author \\ Dr P.S. Srinivas, M.S (Gen) \\ Associate Professor, ACSR Medical College, Nellore (A.P), INDIA
}

\section{Aim of the Study}

1. To study the incidence of various foot lesions in diabetic foot patients attending to Malla Reddy Institute of Medical Sciences, Suraram $\mathrm{X}$ roads, Quthubullapur, Telangana

2. To study the role of contributory factors in the initiation and perpetuation of the ulcer.

3. To study the role of Hospital admission and aggressive surgical management in the treatment of diabetic foot ulcers.

\section{Introduction}

Diabetes mellitus is highly prevalent in India, with large share of diabetic persons called the "Diabetic Capital of the World" India and has an uphill task to care for its wide population. There were 31.7 million in 2000 cases of Diabetes in India and the figure is expected to rise further to 79.4 million cases by year $2030 .^{1}$ The earliest manifestation of Diabetes, Tabes, Pernicious Anemia, Polycythemia, Metabolic disorders including Gout as well as vascular, Brain and spinal cord disorders are often seen in the foot. This is particularly true of Diabetes.

Diabetic foot is disorders such as ulceration, infection, and gangrene are the leading causes of hospitalization in patients with disability, recurrent infections and premature mortality. ${ }^{3}$

Approximately $15 \%$ of patients will develop foot ulcer, about $14-24 \%$ of them may require an amputation. ${ }^{4}$ The annual prevalence of diabetic foot ulcers $6.3 \%$ in US. ${ }^{5}$ India prevalence of foot ulcer is $2.1 \%$ to $21.4 \%$. The risk of Lower Extremity Amputation (LEA) rate is 15 to 46 times higher in diabetics versus non diabetic people. ${ }^{6}$

Clinical studies show that ulcers precede $85 \%$ of non-traumatic lower extremity amputations among diabetic patients. ${ }^{7,8}$ Early detection and appropriate treatment of these ulcers may prevent large number of these amputations.

In developing countries, the diabetic foot represents a major cause of morbidity, mortality, and involves enormous treatment costs. It is thus necessary that the 'high risk' foot in persons with diabetes be identified and treated appropriately by 
increasing awareness, education, blood glucose control, and maintaining a constant vigil for minor insults that have the potential to develop into major problems if not detected and treated in time. Agents that demonstrate clinical effectiveness in the treatment of the infected diabetic foot: ${ }^{34}$

i) Cephalosporin's (Cephalexin orally; cefoxitin and ceftizoxime parenterally)

ii) Penicillin/B-lactamase inhibitor congeners (amoxicillin/clavunate orally; Ampicillin/ Sulbactum, Piperacillin/Tazobactum, and ticarcillin/ clavunate parenterally)

iii) Fluoroquinolones (ciprofloxacin orally and parenterally; ofloxacin orally and parenterally; levofloxacin orally and parenterally)

iv) Other agents
Clindamycin $(\mathrm{O} \& \mathrm{P})$
Imipenem/cilastin (P)
Linezolid (O \& $\mathrm{P})$

Investigations: 1.Fasting and Post Lunch Blood Sugar (FBS \& PLBS): 2.Blood Urea 3.Serum Creatinine: 3:Complete Blood picture,4:Lipid profile, 5:BT,CT, 6:E.C.G, 6: Swab for Culture, 7:X Ray Foot<, Chest, CT, MRI, Doppler, Angiography, Nucleotide Scanning

\section{Management of Diabetic Foot}

\section{A. Medical Management}

Control Of Diabetes: Patient Education, Dietary modification, Physical activity, Pharmacological therapy, Treatment of DKA

Patient Education: Goes a long way in the accurate control of diabetic status.

Dietary Modification: Composition and timing of meals are particularly important.

Caloric goal about 35k.Caloris/ kg/day. Carbohydrate: $60-5 \%$,

Protein $10-20 \%$, Fats : $25-35 \%$.Use of alcohol is forbidden strictly.

Physical activity: 20min walking three times a week has proven a beneficial effect.

Pharmacologic therapy: All patients must be put on soluble (Regular) insulin therapy. Surgical problems with high insulin requirements:
1. Sepsis, infections, abscess, cellulites, fasciitis, burns

2. Obese surgical patients

3. Vascular occlusions or gangrene

4. Diabetic ketoacidosis (DKA)

5. Total parenteral nutrition

6. Glucocorticoid therapy

Insulin Therapy

1. Administer insulin, half the usual morning dose is given subcutaneously as Neutral Protamine Hagedorn (NPH).

2. Start an infusion of $10 \mathrm{dl} 5 \%$ dextrose normal saline solution at $1 \mathrm{dl} / \mathrm{hr}$.

3. During the operation, a separate intravenous line for IV fluids may be needed.

4. Monitor blood glucose levels intra operatively during long operations, in recovery room, and four times daily thereafter.

5. If the blood glucose level is greater than $250 \mathrm{mg} / \mathrm{dl}$ give $10 \%$ to $15 \%$ of the total daily insulin requirements as regular insulin subcutaneously.

6. Give a second dose of NPH half the usual dose, 8-12 hours later.

7. Continue administering intravenous dextrose 5\% solution with $20 \mathrm{mEq}$. Of potassium chloride. Total daily dextrose intake is $150 \mathrm{~g}$ or more. Potassium chloride additions are based on potassium monitoring.

8. A split dose of NPH should be continued daily after surgery. Add regular insulin if the blood glucose level is elevated.

9. For unstable patients, change to the dextrose insulin infusions method or to separate intravenous insulin infusion.

10. Resume the usual insulin regimen when oral feeding has started.

1. Schedule surgery in the morning.

2. Start an infusion of $10 \mathrm{dl} 5 \%$ dextrose in $0.9 \%$ sodium chloride (DNS) at $1 \mathrm{dl} / \mathrm{hr}$ with regular insulin, infusion rate may be 
increased during the operation if the fluid requirements are elevated.

4. Check the blood glucose level in the recovery room and four times daily thereafter.

5. Adjust the insulin dose based on the blood glucose levels, Add potassium chloride, $20 \mathrm{mEq}$. to infusions post operatively. If serum potassium level $<3.5$, increase potassium chloride to 40 to 80 mEq./L., if serum potassium level $>5$, no potassium chloride is added.

1. Post operative management: Administer dextrose insulin potassium infusion at rate to maintain hydration usually $3 \mathrm{~L}$ daily in half normal saline solution; add multivitamin preparation. Adjust insulin and potassium chloride based on daily monitoring data.

2. Resume subcutaneous insulin administration on recovery; stabilize the patient with multicomponent regimen.

Glucose - insulin infusion for non- insulin dependent diabetics:

1. On the day of surgery, start the infusion of 10 dl 5\% dextrose in normal saline solution with $8-10$ units of human regular insulin at $1 \mathrm{dl} /$ $\mathrm{hr}$.

2. Monitor the plasma glucose level in recovery room and four times daily thereafter.

\section{Blood glucose level}

$<90 \mathrm{mg} / \mathrm{dl}$
$90-199$
$200-280$
$>280$

$<90 \mathrm{mg} / \mathrm{dl}$

$>280$

3. Add potassium chloride $(20 \mathrm{mEq})$ to the dextrose - insulin infusion post operatively.

4. Continue the dextrose - insulin -potassium infusion until oral feeding is resumed. Adjust regular insulin and potassium chloride additions based on monitoring data.

5. Insulin may be required for variable periods during recovery before resuming sulfonylurea or diet alone.
At discharge from hospital, when we contemplate on reinstituting dietary therapy and or oral hypoglycemia drugs for diabetic control.

It is customary to keep these patients in hospital for this regimen for at least a week for adequate stabilization. Never discharge a patient after abruptly shifting him from insulin therapy to diabetic or oral drug management as it may trigger sudden deterioration.

\section{Insulin Replacement}

Initially started with a loading dose of 0.3 units $/ \mathrm{kg}$ as a bolus to prime tissue insulin receptors followed by $0.1 \mathrm{unit} / \mathrm{kg} / \mathrm{hr}$; either continuously infused or given intramuscularly. In insulin resistance the dose is doubled every 2-3 hrs, if hyperglycemia doesn't improve.

\section{Control of Infection}

Achieved by using a combination of Inj. ceftriaxone $1 \mathrm{gm} / \mathrm{IV} / \mathrm{BD}$ along with Inj. metronidazole $800 \mathrm{mg} / \mathrm{IV} / \mathrm{TID}$. In severe cases, Inj. Piperacillin + Tazobactum 4.5 gm/ IV / TID .

\section{General Supportive Care}

a) Tab. Pentoxyphylline 400mg / PO / TID for $8-10$ weeks.

b) Vitamins with a balanced caloric diet.

c) Correction of anemia by blood transfusions

\section{B. Surgical Management}

\section{Treatment of Corns and Calluses}

Wear proper fitting shoes. Excess keratosis removed by soaking the feet in lukewarm soap water for $10-15$ minutes, then rub off the excess tissue with a towel. Do not tear it off.

Excision and deroofing can be done and granulation scraped if there is infection.

2. Treatment of Fissures: Fissures treated with regular applications of moisturizing and/or antifungal creams like Eucerin, hydrated lanoline, antifungal like Tolnaftate, especially between the toes.

3. Treatment of Blisters: Blisters must be opened or unroofed to relieve the pressure on the underlying tissue and to permit culture of the 
Exudate. These lesions should then be treated with an antibiotic ointment such as Neosporin or Betadine.

4. Local Wound Care: protecting the wound from trauma and contamination, to facilitate wound drainage and to provide antibacterial coverage . The primary goal in the treatment of diabetic foot ulcers is to obtain wound closure.

5. Minor Ulcers: A dry gauge pad changed once or twice daily is adequate.

Topical medications:
a. 5\% Povidone Iodine solution,
b. $1 \%$ Silver sulphadiazine cream
c. Gentamycin cream, Bacitracin ointment
d. Hydrogen peroxide

warm environment provided by a number of commercially available special dressings, including semi permeable films, foams, hydrocolloids, and calcium alginate swabs. ${ }^{42}$

6. Major Ulcers: Moderate to severely infected lesions needs hospitalization and adequate debridement.

Methods: Superficial debridement, Incision and drainage of abscesses Debridement of deep infection and necrosis.

\section{Principles to be followed during a debridement}

i. All areas of dead tissue should be removed.

ii. The margins of the ulcer should be trimmed up to the point, where it bleeds freely.

iii. An open wound particularly near a joint should be probed to see for communication.

iv. If necrosis of the skin of the dorsum of the foot follows cellulites, then Radical debridement including the dorsal skin is necessary.

v. In case of central plantar space abscesses, the entire plantar surface should be drained along with amputation of the necrotic toes.

vi. It should be remembered that loss of plantar fascia flattens the longitudinal arch. Special footgear may be needed later to accommodate the new pattern of weight bearing.

vii. Tissues left behind during debridement should not be extensively handled by forceps or any other crushing instruments. viii. fluid or pus is should be sent for culture \& sensitivity test.

Debridement types- Autolytic, Mechanical, Surgical and Enzymatic.

\section{Treatment of Neuropathic Ulcers}

The main aim in treating neuropathic ulcers is to reduce the mechanical stress and pressure of the insensitive feet while maintaining ambulation, by giving rest (Off - loading), elevation of the affected foot, and relief of pressure.

Treatment of More Severe Infections: Limbthreatening infections can be defined by Cellulitis extending beyond $2 \mathrm{~cm}$ from the ulcer perimeter, as well as deep abscess, osteomyelitis, or critical ischemia. $^{44}$

Polymicrobial infections predominate in severe diabetic foot infections and include a variety of aerobic gram-positive cocci, gram-negative rods, and anaerobes. ${ }^{45,46}$ Surgical drainage, deep debridement, or local partial amputations are necessary adjuncts to antibiotic therapy of infections that are deep or limb threatening. ${ }^{47}$ In case of improvement in the general condition, then conservative excision of toes and at the same time the plantar spaces are opened up by longitudinal incisions with division of plantar fascia.

When pus is present, flexor tendon sheaths should be opened up, and pus is drained, sent for Culture and Sensitivity. Post operatively the wounds are inspected daily and $\mathrm{H}_{2} \mathrm{O}_{2}$ irrigation is carried out and dressing with antiseptic solution daily should be made (e.g. Betadine)

\section{Reconstructive Arterial Surgery}

Reconstructive arterial surgery should be considered at this stage after determining the ankle systolic pressures by Doppler flow meter. If the ankle pressure is less than $2 / 3$ of the arm pressure there is very significant large vessels disease under such circumstances reconstructive arterial surgery for limb salvage is worth attempting.

The surgery carried out will depend on the extent of arterial occlusion demonstrated on an arteriogram. The criteria are that there must be an 
adequate pressure above the area to be constructed and there must patent vessels distal to reconstruction so that the increased blood flow can be distributed to the peripheral tissues.

\section{Indications for Peripheral Vascular Surgery in the Diabetic Foot}

a. Nocturnal pain, Rest pain ,Foot ulcers that do not respond to treatment.Infections that do not respond to treatment ,Incipient gangrene

b. Severe disabling intermittent claudication (in select cases)

Thus arterial surgery should be considered if a patient with arterial insuffienciency has:

1. Claudication which significantly interferes with work or worthwhile recreational activities

2. Rest pain

3. An area of gangrene which doesn't heals or cannot be removed surgically, treated with success by a local foot operation. Operation is not indicated in mild claudication to prevent more serious trouble.

Non Operative Correction of Selected Arterial Blockages: Angiography

Transluminal balloon dilatation is done by means of a small balloon catheter which is carefully introduced into site of stenosis and distended under fluoroscopy, thereby relieving the obstruction. Sympathectomy has no role to offer except in young diabetics with peripheral vascular disease whose foot can be demonstrated to have ability to sweat.

\section{Minor Amputations}

The principles governing local amputations are:

i. Infected areas must be opened widely

ii. Tourniquet is to be avoided

iii. There is no place for small incision of fluctuant areas and insertion of drainage tubes through the foot. There is usually deep infection which requires wide incisions and excision of the devitalized tissues. iv. The other foot must be taken care of because this may be the only one the patient will have.

\section{Various Types of Local Amputations}

1. Transphalangeal amputation of a toe

2. Amputation of a single toe and Ray amputation

3. Transmetatarsal amputation

4. Syme's amputation

\section{Transphalangeal Amputation of a TOE}

This is the most commonly performed amputation. If there is evidence of arterial insufficiency, adequate collateral circulation as shown by a venous filling time of 20 seconds or less is necessary. The lesion must be in the distal $1 / 3^{\text {rd }}$ of the toe, leaving reasonably healthy skin at the site of incision. There should be no dependent rubor of the proximal part of the toe and all Cellulitis and lymphangitis should have cleared prior to operation. To accomplish these ends, up to 3 weeks In patient hospital care may be required with few exceptions the aim is to be able to do a closed procedure since open amputations in the presence of diminished circulation do not do well. The skin edges are approximated with minimally reactive sutures and the sutures are left in place for up to 4 weeks. Delicate handling of the skin flaps with avoidance of forceps is mandatory for a successful result.

\section{RAY Amputation}

This operation removes one toe and distal half metatarsal shaft including the head through a racquet shaped incision, this leaves a residual space which cannot be surgically closed and heal by secondary intention with scar formation which eventually pulls the adjacent metatarsal together. The adjacent joint capsules are avascular structures which further delay healing; the operation is therefore reserved for those patients with quite good collateral circulation. This procedure is more useful in the neuropathic rather than the ischemic foot and hence mainly done for ulcers under the first or fifth metatarsal heads or beneath one of the other metatarsal head. Usually the incision is closed over its distal $80 \%$ and their 
proximal racquet handle is left open for dependent drainage. For the first and fifth toes the proximal incision is carries along the lateral or medial edge of the foot off the weight bearing areas.

\section{Transmetatarsal Amputation}

Transmetatarsal amputation may have to be done if more than one toe is involved or there is persistent recurrent plantar ulcer. In this, one removes all of the toes and metatarsal heads, using a plantar flap resembling a Turkish slipper for closure.

Infection must be controlled and the skin of the dorsum and sole of the foot must be healthy. It may take up to 3 weeks of care in the hospital to prepare the patient for the operation. Pulses need not be present in the foot but the collateral with a venous filling time of less than 25 seconds and absence of dependent rubor at the level of incision. With the patient under SA or GA, fairly straight dorsal incision is made slightly proximal to the metatarsal heads. After surrounding the bone ends to give a smooth, contour to the foot, closure is made with one layer of non-reactive sutures on the whole the results are not satisfactory.

SYME'S Amputation: This is indicated where more than one toe is involved, sepsis is confined to distal half of the foot and the large vessel disease is absent. The role of Syme's amputation is diabetic foot however still remains controversial. The main reason for its advocacy is that the patient will be able to walk on his own leg (feeling of earth) and without shortening of his limb.

\section{Major Amputation}

Usually it is the ischemic diabetic foot, with significant large vessels disease with gangrene which requires a major amputation. The level of major amputation poses a difficult problem as to whether it should be above or below knee. The above knee healing is almost certain but the knee should be sacrificed. Also when the general condition is very poor and the patient is a severe diabetic state, when the infection and toxemia are not controlled, major amputation cannot be avoided.

Below Knee Amputation: We select the below knee level if the patient is expected to use a prosthesis, if the area of gangrene is below the ankle with a demarcation at or below the ankle level. Walking with prosthesis is definitely more satisfactory following this procedure than after higher amputation.

The amputation is usually accomplished slightly below the mid lower leg level using short and equal anterior or posterior flaps, dividing the fibula one inch higher that the tibia, and the tibia at a suitable level to permit easy closure. Occasionally when there is scarring of the skin over the tibia or the leg is thin, medial and lateral flaps are used.

Post operative splint is worn for one week to prevent knee contracture. Stump can be fitted with prosthesis near the $14^{\text {th }}$ post operative day.

Above Knee Amputation: The operations most commonly performed are:

a) Through knee disarticulation (GrittiStokes)

b) Supra condylar amputation

c) Mid - thigh amputation

The first two operations are likely to heal well due to the presence of collateral vessels in the area of the knee joint and the subcutaneous tissues. Both operations involve divisions of only small amount of muscle tissues which has the practical advantage of reduced anaerobic sepsis. Both have a disadvantage of difficulty in fitting a proper prosthesis. Of the three the supra condylar amputation offers the ideal stump.

\section{Prevention (Diabetic Foot Care)}

Prevention against development and progression of minor foot lesions in diabetics remains the main target. ${ }^{48}$ major and severe lesions require surgical intervention.

Care of the foot takes place at three levels

i) The patient must take routine measures to care of his feet.

iii) Advanced lesions require specialist surgical care. 
The best approach is to make use of a team of multidisciplinary professionals. Physician, Nurse, Endocrinologist, Podiatrist, Neurologist, Vascular surgeon, Orthopedist, Physiotherapist, Social workers

Education of the patient is an essential role

1. As pain sensations are diminished, the patient must be trained to utilize his other sense to avoid injury and if there occur. patient must seek advice if any swelling, cracks, soreness or redness are present. Never allow them to walk barefoot. Daily washing / changing of socks is advised.Calluses and corns are treated with great care.

2. Elective surgery to correct structural deformities needed in certain patients.

\section{Materials and Methods}

All patients with diabetes who presented with foot lesions to MALLA REDDY INSTITUTE OF MEDICAL SCIENCES (TELANGANA) in outpatient between "2009 - 20011" were included in this study.

After enrolment, a detailed clinical history was taken. This is followed by a detailed examination of the patient with particular reference to the lesion of the foot.

The presence of associated arterial disease was determined by a clinical examination of the lower limb pulses. The presence of neuropathy was ascertained by means of a monofilament test.

Patients who had associated medical complications, poor control of diabetic and/or foot ulcers Grade II and above were selected for inpatient hospital management.

Baseline work up of patients included a Complete blood count, Urine examination, FBS and PLBS, Blood urea, Creatinine, Lipid profile, ECG, Chest X-ray, X- ray of foot.

Patients with diabetes for greater than 5 years and patients who were symptomatic were screened for retinopathy by means of Fundus examination.
Medical treatment was directed towards obtaining a Euglycemic status and maintaining it. All ulcers are inspected daily. Patients with Grade 0 and I were inspected in the outpatient department. Healthy ulcers were cleaned with NS and dressed. Ulcers with slough and ulcers whose extent of infection could not be determined clinically were subjected to surgical treatment which included Excision of all the necrotic tissue and laying open of all infected planes. The wounds were subjected to a thorough wash using Normal saline and Hydrogen peroxide and dressed with Betadine. Patients whose ulcers healed were instructed on foot care and discharged. Those who had a large raw area after healing of ulcer were sent for a plastic surgical consultation and appropriate grafting procedures were done whenever feasible. Patients whose infection failed to respond to treatment or with obvious gangrene were subjected to amputation at the appropriate level.

\section{Analysis}

During the period of study from 2009-20011, a total of 62 cases were enrolled and included in the study. They were managed by different modalities of treatment both medical and surgical, after which they were followed up for different length of time.

A birds eye view of the incidence of various lesions and response to treatment adopted, the failure and success rates has been depicted in the form of tables to enable the reader to assess, and explain the treatment and prognosis and results by different modes of treatment. In this study Total No. of patients 62 , the No. of male patients 36 and female 26. 

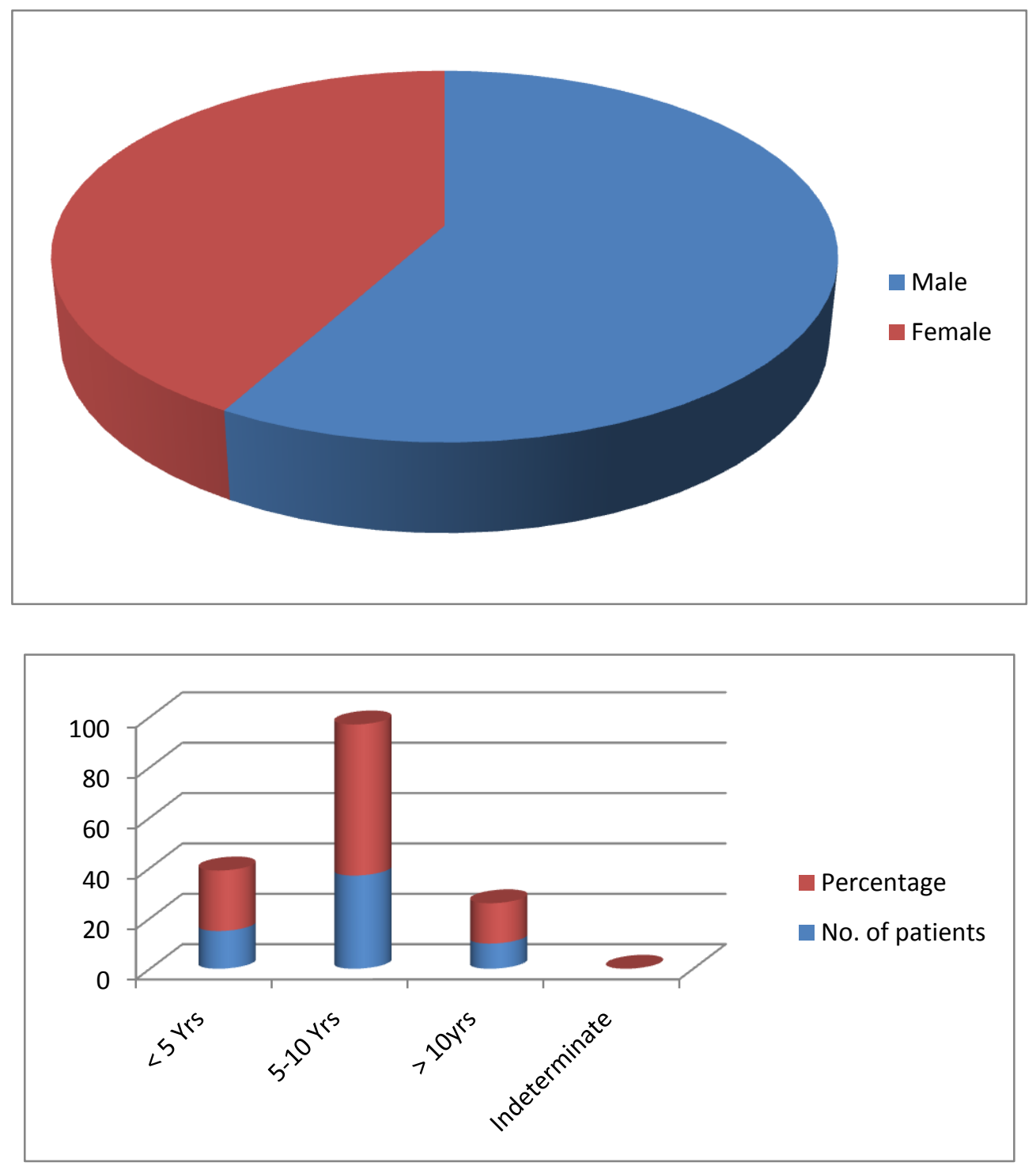

Most of the patients presented with severe disease to the hospital

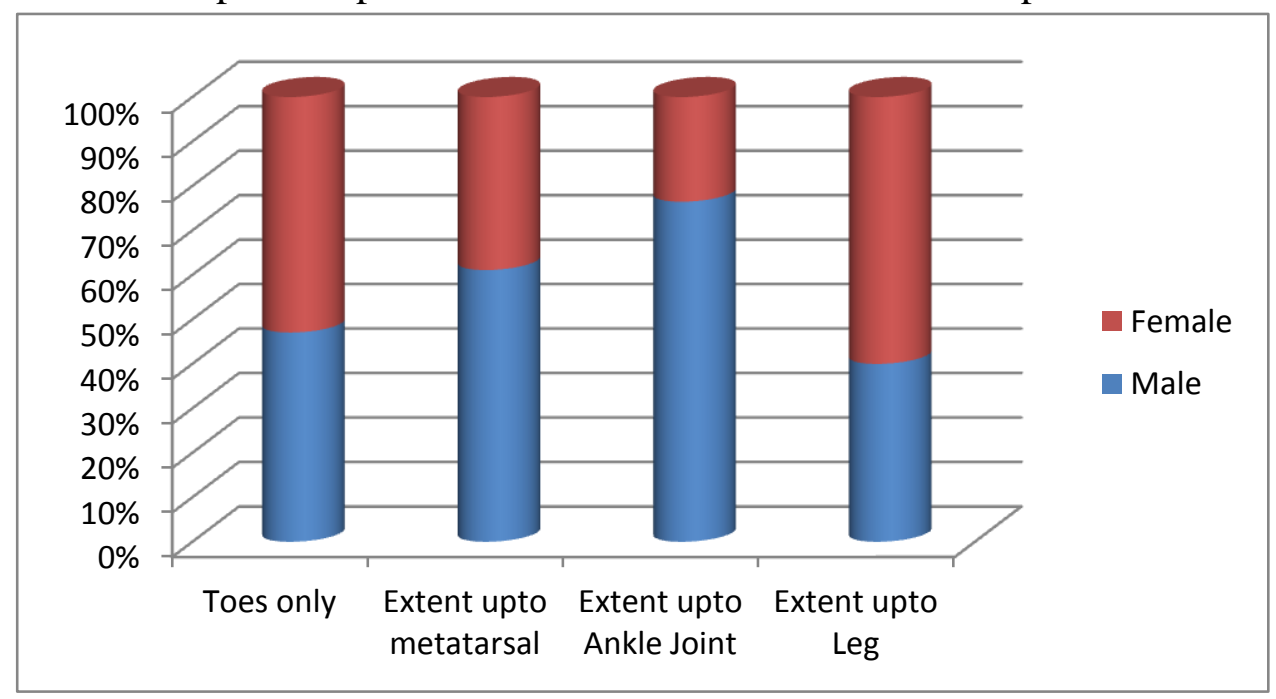


Distribution of Clinical Presentation of Diabetic Foot and Management Required in 62 Cases

\begin{tabular}{|c|c|c|c|c|c|}
\hline & $\begin{array}{c}\text { BULLAE } \\
\text { FORMATION } \\
\& \\
\text { ULCERATION } \\
\end{array}$ & $\begin{array}{c}\text { TROPHIC } \\
\text { ULCER }\end{array}$ & \begin{tabular}{|c|} 
TROPHIC \\
ULCER \\
CHARCOT \\
JOINT \\
\end{tabular} & \begin{tabular}{|c|} 
ULCER \\
CELLULITIS \\
OEDEMA \\
\end{tabular} & $\begin{array}{c}\text { GANGRENE OF } \\
\text { TOES WITH } \\
\text { CELLULITIS }\end{array}$ \\
\hline Total & 7 & 5 & 3 & 22 & 15 \\
\hline Drug therapy & 7 & 5 & 3 & 22 & 15 \\
\hline Wound toilet & 7 & 5 & 3 & 22 & 15 \\
\hline $\begin{array}{l}\text { Wound debridement } \\
\text { and skin grafting }\end{array}$ & 5 & 3 & 3 & 12 & 2 \\
\hline \multicolumn{6}{|l|}{ Minor Amputation } \\
\hline a. Toe & 2 & 2 & & 6 & 5 \\
\hline b. Transmetatarsal & & & & 1 & 1 \\
\hline \multicolumn{6}{|l|}{ Major Amputation } \\
\hline a. $\mathrm{B} / \mathrm{K}$ & & & & 3 & 6 \\
\hline b. A/K & & & & & 2 \\
\hline
\end{tabular}

\section{1, Amputations}

\begin{tabular}{|c|c|}
\hline Type of Amputation & Number of Patients \\
\hline Minor & 20 \\
\hline Major & 13 \\
\hline
\end{tabular}

In this study $53 \%$ of patients required amputations in which minor in $32 \%$ patients and major in $21 \%$ patients.

\section{Discussion}

Diabetic patients have always suffered from complications affecting the lower limbs. Ulceration, Foot infection and the subsequent amputation of a lower extremity are the most common cause of hospitalization among diabetic patients.

As seen in our study, Agriculturists had more incidences of diabetic foot lesions because of ignorance of having diabetes, lack of foot care and poor management of diabetes.

Conforming with major studies worldwide, diabetic foot ulcers have been more in the patients with NIDDM (87\%)

Neuropathy which is seen in more than $50 \%$ of diabetics of long- standing duration (Pirat 1978) is considered at be the single most major cause of ulcerations in the diabetic foot. In this study Neuropathy was seen in $61 \%$ of the patients.

It is also clearly seen that the duration of diabetes mellitus had an impact on the foot lesions. Patients had ulcer with diabetes of more than 5 years was seen in $52 \%$ of cases. It has been shown in the epidemiological surveys of diabetic foot problems in the Stockholm Country, the foot ulcers were found in about $54.9 \%$ of patients who had diabetes for more than 5 years.

This is mainly because the ulcer is related to the occurrence of neuropathy or vasculopathy which are long-term complications of this disease.

Wagner's classification score may be different for a surgeon as compared to physician because they come with advanced disease to a surgeon and for this reason patient with grade 0,1 and 2 are lesser and those with grade 3,4 and 5 are more in our study.

The standard treatment for diabetic foot according to Wagner's classification is Prevention for grade0 , Antibiotics and good glycemic control for grade 1. In grade-2 needs hospital admission, as they need surgical intervention along with antibiotics and glycemic control. Grade- 3 requires some sort of amputation, In grade- 4 wide debridement and amputation while in grade-5 the preferred treatment is below knee amputation.

Daily surgical debridement is the mainstay in the treatment of diabetic ulcers was carried out in nearly $80 \%$ of the patients. $85 \%$ of patients had good healing, $15 \%$ of patients had poor healing. $14 \%$ of patients needed skin grafting. Around $30 \%$ of patients had undergone some form of amputation minor or major in the management of severe diabetic foot lesions.(Wagner grade III-V). 


\section{Conclusions}

A patient of diabetes mellitus is exposed to a number of complications of which Diabetic foot is a common condition. The surgeon is usually facing the problem of "Life and Leg". With proper understanding of the disease and the new concepts in the management of Diabetic foot, it has been possible to save many limbs and lives.

This study comprised of 62 cases of diabetic foot patients with emphasis on surgical management and its complications over a period of 18 months. After analysis of the data the following are the conclusions.

- The youngest patient was 40 years old and the oldest 90 years. The highest number of patients was seen in the age group of 51-60 years $(32 \%)$.

- Males are almost three times more affected than females. (Males are more vulnerable to trauma).

- Duration of diabetes varied from 1year to 25 years and few patients were diagnosed post admission.

- Trivial trauma of some kind was the initiating factor in nearly half of the cases.

- Minimum stay in hospital was 1 week and maximum 96 days.

- Commonest presenting lesion was Ulcer $56 \%$, followed by Cellulitis 20\%, and Gangrene 20\%.

- Conservative treatment consisting of control of diabetes with Plain/Lente insulin along with appropriate oral / IV antibiotics was effective in some cases.

- Wound debridement, slough excision followed by dressing with Povidone/ magnesium sulphate/ framyctine /metrogyl/ collagenese dressing resulted in healing in some cases.

- Split skin grafts, Disarticulation, Transmetatarsal amputation, below knee and above knee amputations were the other modes of treatment.

Early presentation and hospital admission, aggressive and appropriate medical and surgical treatment according to grade of disease can improve outcome and reduce the morbidity and mortality due to diabetes.

\section{Summary}

Foot ulcers are one of the major complications of diabetes. They have a poor tendency to heal, which may result in long stay in hospital for treatment. The foot in diabetic patient is the cross road of several pathological processes, in which almost all components of the lower extremity are involved- skin, subcutaneous tissue, muscles, bones, joints, blood vessels and nerves. An understanding of the development of complications and application of preventive and management strategies will reduce the complications of diabetic foot. Patients who are diabetic should take proper care of their feet so that they will not suffer from the consequences. They should take proper medical care at the earliest once any trauma occurs, so that the chain of events could be halted. With proper care by the attending surgeon which includes regular dressing, wound debridement, proper antibiotics the outcome can be favourable and avoid the unwanted surgeries like amputations.

\section{Bibliography}

1. Wild S, Roglic G, Green A, Sicree R, King H. Global prevalence of diabetes Estimates for the year 2000 and projections for 2030 . Diabetes Care 2004;27:1047-53

2. Boulton AJ. The diabetic foot: a global view. Diabetes Metab Res Rev 2000;16 (suppl 1):s2-5.

3. Frykberg RG, Armstrong DG, GIurini J, Edwards A, KRavette M, Kravitz S, et al. Diabetic Foot disorders: a clinical Pracice guideline. American college of Foot and Ankle Surgeons. J Foot Ankle Surg 2000;39 (5 suppl):s1-60.

4. Reiber GE, Boyko EJ, Smith DG. Lower extremity foot ulcers and amputations in diabetes; In: National Diabetes Data Group (U.S.). Diabetes in America.2d ed. 
Bethesda, Md.: National Institutes of Health, National Institute of Diabetes and Digestive and Kidney Diseases, 1995; NIH publication no.95-1468.

5. Resource from National Institute of Health (NIH) American Diabetes Association. Standards of medical care in diabetes -2010. Diabetes Care. 2008 Jan;33 Suppl 1:S11-61.

6. Lavery LA, Ashry Hr, van Houtum W, Pugh JA, Harkless LB, Basu S. Variation in the incidence and proportion of diabetes-related amputations in minorities. Diabetes Care 1996;19:48-52

7. Pecoraro RE, Reiber Ge, Burgess EM. Pathways to diabetic limb amputation. Basis for prevention. Diabetes care 1990;13:513-21.

8. American Diabetes Association. Consensus Development Conference on Diabetic Foot Wound Care: 7-8 April 1999, Boston, Massachussets. Diabetes Care 1999;22:1354-60.

9. Sumpio BE. Foot ulcers. NEJM 2000;343:787-93.

10. Stevens Mj, Feldman El, Greene DA: The aetiology of diabetic neuropathy: The combined roles of metabolic and Vascular defects. Diabet Med 12:566,1995.59.

11. Wilson RM. Neutrophil function in diabetes. Diabet Med 1986;6:509-12..

12. McMahon MM, Bistrian BR. Host defences and susceptibility to infection in patients with diabetes mellitus.Infect Dis Clin North Am 1995;9:1-10.

13. Sentochnik De, Eliopoluos GM. Infection and Diabetes. In, Joslin's Diabetes Mellitus, $13^{\text {th }}$ edition.CR Kahn and GC Weir, eds. Lea \& Febiger (Philadelphia) 1994. Pages 867-68.

14. Bridges RM, Deitch EA. Diabetic foot infections. Pathophysiology and treatment. Surg Clin North Am 1994;74:537-635.
15. Lipsky Ba. Infectious problems of the foot in diabetic patients. In, the Diabetic Foot, $6^{\text {th }}$ edition., J.H. Bowker, M.A. Pfeifer, eds, St. Louis, Mosby, 2001, pages 46780.

16. Bokyo EJ, Ahoroni JH, Stensel V, Forsberg RC, Davignon DR, Smith DG. A prospective study of risk factors for diabetic foot ulcer: the Seattle Diabetic Foot Study. Diabetic Care 1999;22:103642.

17. Reiber GE, Vileikyte L, Boyko EJ, del Agulia M, Smith DG, Lavery LA, et al. Causal pathways for incident lowerextremity ulcers in patients with diabetes from two settings. Diabetes Care 1999;22:157-62.

18. Armstrong Dg, Lavery La. Diabetic foot ulcers:prevention, diagnosis and classification. Am Fam Physician 1998;57: 1325-32.

19. McNeely MJ, Boyko EJ, Ahroni JH, Stensel VL, Reiber GE, Smith DG, et al. The independent contributions of diabetic neuropathy and Vasculopathy in diabetic foot ulceration. How great are the risks?Diabetes Care 1995;18:216-219

20. Grayson ML, Gibbons GW, Balogh K, Levin E, Karchmer AW.Probing to bone in infected pedal ulcers: a clinical sign of underlying osteomyelitis in diabetic patients. JAMA 1995;273:721-32. 